1] Prace Matematyczno-Przyrodnicze 4, Shupsk 1983, 3-12.

[2] Z. Grande, Sur les fonctions approximativement quasi-continues, Revue Roum. Math. Pures et Appl, à paraître.

3] - Sur la continuité approximative faible, Problemy Matematyczne 4 (1984), 11-18.

[3] - Sur la continuile approgie I, Warszawa 1958.

[5] J. Oxtoby, Measure and category, New York-Heidelberg-Berlin 1971.

[6] D. Preiss, Limits of approximately continuous functions, Czech. Math. J. 96 (1971), 371-372.

\section{INSTYTUT MATEMATYKI}

WYŻSZA SZKOEA PEDAGOGICZNA

Bydgoszcz
Received 31 December 1985;

in revised form 20 October 1986

\section{Strongly discrete subsets in $\omega^{*}$}

R. Frankiewicz (Gliwice) and P. Zbierski (Warszawa)

Abstract. We prove that the statement: " $\bar{D}=\beta D$ for each strongly discrete subset $D \subseteq \omega^{*}$ with $|D|=\omega_{1}$ " is consistent with ZFC+MA. We also give an example of a $B$-ideal over $\omega$ which cannot be extended to a $P$-point.

0. It is well known that if $D$ is a countable discrete subset of the remainder $\omega^{*}=\beta[\omega] \backslash \omega,(\beta[\omega]=$ the Stone-Cech compactification of the discrete space $\omega)$, then the closure $\bar{D}$ in $\omega^{*}$ is (homeomorphic to) the space $\beta[\omega]$, or equivalently, $D$ is $C^{*}$-embedded in $\omega^{*}$.

In this paper we turn our attention to discrete sets $D \subseteq \omega^{*}$ of cardinality $\omega_{1}$. Under the consistent assumption $2^{\omega_{0}}=2^{\omega_{1}}$, the space $\beta\left[\omega_{1}\right]$ (the Stone-Cech compactification of a discrete space of cardinality $\omega_{1}$ ) can be embedded into $\omega^{*}$. Hence we may ask whether $\bar{D}=\beta D$ for discrete $D$ with $|D|=\omega_{1}$.

Balcar, Simon and Vojtáš [1981] constructed a discrete set $D \subseteq \omega^{*},|D|=\omega_{1}$ having the following property: there is a point $x \in \omega^{*}$ such that each neighbourhood of $x$ contains all but countably many points of $D$. Obviously, $\bar{D} \neq \beta D$ for such a $D$. Hence we shall consider strongly discrete $D$ in the following sense: there is a family of pairwise disjoint closed-open neighbourhoods, each containing a single point of $D$. Note that each countable discrete set $D$ is strongly discrete.

The main result of this paper is the following

THEOREM. Assuming the consistency of the Zermelo-Fraenkel set theory ZFC, there is a model of ZFC plus Martin's Axiom in which the closure $\bar{D}$ of each strongly discrete set $D \subseteq \omega^{*},|D|=\omega_{1}$, is homeomorphic to $\beta D$ (i.e. $D$ is $C^{*}$-embedded in $\omega^{*}$ ). In addition, $2^{\omega_{0}}=\omega_{2}$ and $\beta\left[\omega_{1}\right]$ is not a continuous image of $\omega^{*}$.

It can be proved, that the theorem fails in a model obtained by adding $\omega_{2}$ Cohen reals.

1. We represent $\beta[\omega]$ as the space of all ultrafilters over $\omega$ with the Stone topology. The remainder $\omega^{*}=\beta[\omega] \backslash \omega$ consists then of all nonprincipal ultrafilters. The basic open-closed neighbourhoods are of the form $A^{*}=\bar{A} \cap \omega^{*}$, for an $A \subseteq \omega$, and $A^{*}$ consists of all nonprincipal ultrafilters containing the set $A$. Let $D=\left\{F_{\alpha}: \alpha<\omega_{1}\right\}$ be a strongly discrete set of cardinality $\omega_{1}$. According to the Taimanov Theorem (Engelking [1968]) in order that $\bar{D}=\beta D$ it is sufficient that 
for an arbitrary $E \subseteq \omega_{1}$, the parts

$$
\left\{F_{\alpha}: \alpha \in E\right\} \quad \text { and } \quad\left\{F_{\alpha}: \alpha \notin E\right\}
$$

can be separated by open-closed subsets of $\omega^{*}$.

Since, by our assumption, $D$ is strongly discrete there are almost disjoint sets $A_{\alpha} \subseteq \omega$ such that $A_{\alpha} \in F_{\alpha}$ for all $\alpha<\omega_{1}$. Consider the following forcing notion $\boldsymbol{P}_{E}\left\{A_{\alpha}\right\}$ : the conditions are pairs $p=\left\langle s_{p}, t_{p}\right\rangle$, where $s_{p}, t_{p}$ are finite functions;

$$
\operatorname{Dm}\left(s_{p}\right) \subseteq E, \quad \operatorname{Dm}\left(t_{p}\right) \subseteq \omega_{1} \backslash E, \quad \operatorname{Rg}\left(s_{p}\right), \operatorname{Rg}\left(t_{p}\right) \subseteq \omega
$$

and

$$
\bigcup_{\alpha \in \operatorname{Dm}\left(s_{p}\right)}\left[A_{\alpha} \backslash s_{p}(\alpha)\right] \cap \bigcup_{\beta \in \operatorname{Dm}\left(t_{p}\right)}\left[A_{\beta} \backslash t_{p}(\beta)\right]=\varnothing .
$$

The ordering on $\boldsymbol{P}_{E}\left\{A_{\alpha}\right\}$ is defined as inverse inclusion. Note that since the $A_{\alpha}$ 's are almost disjoint, for arbitrary finite domains

$$
a=\left\{\alpha_{1}, \ldots, \alpha_{n}\right\} \subseteq E \quad \text { and } \quad b=\left\{\beta_{1}, \ldots, \beta_{m}\right\} \subseteq \omega_{1} \backslash E,
$$

any $s, t$ on $a, b$, respectively, form a condition $p=\langle s, t\rangle$ if only the values of $s, t$ are large enough.

Let $G \subseteq \boldsymbol{P}_{E}\left\{A_{\alpha}\right\}$ be a generic filter and let

$$
\begin{aligned}
& s_{G}=\bigcup\{s: \exists t[\langle s, t\rangle \in G]\}, \\
& t_{E}=\bigcup\{t: \exists s[\langle s, t\rangle \in G]\} .
\end{aligned}
$$

Obviously, $s_{G}: E \rightarrow \omega$ and $t_{G}: \omega_{1} \backslash E \rightarrow \omega$. Now, if $A=\bigcup_{\alpha \in E}\left[A_{\alpha} \backslash s_{G}(\alpha)\right]$ and $B=\bigcup_{\beta \notin E}\left[A_{\beta} \backslash t_{G}(\beta)\right]$, then for all $\alpha \in E$ we have $A_{\alpha} \subseteq{ }_{*} A\left(X \subseteq_{*} Y\right.$ denotes "almost inclusion", i.e. $X \backslash Y$ is finite) and $A_{\beta} \subseteq_{*} B$ for $\beta \notin E$ and $A \cap B=\varnothing$. Thus the openclosed neighbourhoods $A^{*}, B^{*}$ will separate the sets $\bigcup A_{\alpha}^{*}$ and $\bigcup A_{\beta}^{*}$ in any model containing $A, B$. After a long enough iteration of separating forcings $\boldsymbol{P}_{E}\left\{A_{\alpha}\right\}$ we obtain an extension $V[G]$ of a ground model $V$ in which property (T) holds for all strongly discrete sets $D \in V[G]$ and hence our theorem will be valid in $V[G]$.

It is obvious that our iteration should not collapse $\omega_{1}$, and even more: at each stage we have to force with a forcing satisfying the c.c.-condition. Generally, a forcing $\boldsymbol{P}_{E}\left\{A_{\alpha}\right\}$ need not satisfy the c.c.-condition: let $\left\{A_{\alpha}: \alpha<\omega_{1}\right\}$ be an almost disjoint family with the property: for each $\alpha<\omega_{1}$ and $k \in \omega$ the set

is finite.

$$
\left\{\beta<\alpha: \max A_{\beta} \cap A_{\alpha}<k\right\}
$$

If both $E$ and $\omega_{1} \backslash E$ are uncountable, then clearly $\boldsymbol{P}_{E}\left\{A_{\alpha}\right\}$ collapses $\omega_{1}$. Nevertheless, for any such $\boldsymbol{P}_{E}\left\{A_{\alpha}\right\}$ we shall find, in the course of iteration, an improvement, i.e. a forcing $\boldsymbol{P}_{E}\left\{B_{\alpha}\right\}$ with $B_{\alpha} \subseteq A_{\alpha}$ which satisfies the c.c.-condition.
2. We shall investigate uncountable antichains in $\boldsymbol{P}_{E}\left\{A_{\alpha}\right\}$. For a condition $p=\left\langle s_{p}, t_{p}\right\rangle$

$$
K_{p}=\bigcup_{\alpha \in \operatorname{Dm}\left(s_{p}\right)}\left[A_{\alpha} \backslash s_{p}(\alpha)\right]
$$

and

$$
L_{p}=\bigcup_{\beta \in \operatorname{Dm}\left(t_{p}\right)}\left[A_{\beta} \backslash t_{p}(\beta)\right] .
$$

Thus $K_{p} \cap L_{p}=\varnothing$. For conditions $p, q$ define

$$
p * q=\left(K_{p} \cup K_{q}\right) \cap\left(L_{p} \cup L_{q}\right)=\left(K_{p} \cap L_{q}\right) \cup\left(L_{p} \cap K_{q}\right) .
$$

If $s_{p} \cup s_{q}$ and $t_{p} \cup t_{q}$ are functions, then $p, q$ are incompatible if and only if $p * q \neq \varnothing$.

Suppose that $\boldsymbol{P}_{E}\left\{A_{x}\right\}$ contains an uncountable antichain. Using the $\Delta$-system lemma and after some thinning out we can assume that there is an antichain $C=\left\{p_{\alpha}: \alpha<\omega_{1}\right\}$ satisfying the following properties:

$p_{\alpha} * p_{\beta} \neq \varnothing$ for all $\alpha<\beta<\omega_{1}$;

$\max \operatorname{Dm}\left(s_{p_{\alpha}}\right)<\min \operatorname{Dm}\left(s_{p_{\beta}}\right)$ for $\alpha<\beta$;

$\max \operatorname{Dm}\left(t_{p_{\alpha}}\right)<\min \operatorname{Dm}\left(t_{p_{\beta}}\right)$;

the domains of all the $s_{p_{\alpha}}$ 's are of the same length: $\left|\operatorname{Dm}\left(s_{p_{\alpha}}\right)\right|=\left|\operatorname{Dm}\left(s_{p \beta}\right)\right|$, for all $\alpha<\beta$ and the same for the $t_{p_{\alpha}}$ 's;

if $\operatorname{Dm}\left(s_{p_{\alpha}}\right)=\left\{\gamma_{1}^{\alpha}, \ldots, \gamma_{n}^{\alpha}\right\}$, then $s_{p_{\alpha}}\left(\gamma_{i}^{\alpha}\right)=s_{p_{\beta}}\left(\gamma_{i}^{\beta}\right)$ for $i=1, \ldots, n$ and all $\alpha<\beta$ and similarly for the $t_{p_{\alpha}}$ 's.

The following lemma states the fundamental property of uncountable antichains.

LEMMA. Let $C=\left\{p_{\alpha}: \alpha<\omega_{1}\right\}$ be an uncountable antichain in $P_{E}\left\{A_{\alpha}\right\}$ as described above. Then there exist a tree $T$ on $[\omega]^{<\omega} \backslash\{\varnothing\}$, an ordinal $\gamma<\omega_{1}$ and a function $q: T \rightarrow\left\{p_{\alpha}: \alpha<\gamma\right\}$ such that for each $\alpha \geqslant \gamma$ there is a branch $e=\left\langle e_{n}: n \in \omega\right\rangle$ of $T$ such that (1) $e_{n}=p_{\alpha} * q(e \mid n)$ for each $n \in \omega$ and (2) the family $\left\{e_{n}: n \in \omega\right\}$ is pairwise disjoint.

Proof. For $e_{0} \in[\omega]^{<\omega} \backslash\{\varnothing\}$ define

$$
X\left(e_{0}\right)=\left\{\alpha>0: p_{0} * p_{\alpha}=e_{0}\right\}
$$

and let $S=\left\{e_{0}:\left|X\left(e_{0}\right)\right|=\omega_{1}\right\}$. Some of the sets $X\left(e_{0}\right)$ can be countable or finite, so take $\gamma_{0}=\sup \left\{\sup X\left(e_{0}\right): e_{0} \in S\right\}$ and write $Y\left(e_{0}\right)=X\left(e_{0}\right) \backslash \gamma_{0}$. We have

$$
\omega_{1} \backslash \gamma_{0}=\bigcup\left\{Y\left(e_{0}\right): e_{0} \in S\right\} \text {. }
$$

Let $q\left(e_{0}\right)=p_{\alpha\left(e_{0}\right)}$, where $\alpha\left(e_{0}\right)=\inf Y\left(e_{0}\right)$. Now, repeat the process for each $q\left(e_{0}\right), e_{0} \in S:$ let

and

$$
X\left(e_{0}, e_{1}\right)=\left\{\alpha \in Y\left(e_{0}\right): p_{\alpha} * q\left(e_{0}\right)=e_{1}\right\}
$$

$$
S\left(e_{0}\right)=\left\{e_{0}:\left|X\left(e_{0}, e_{1}\right)\right|=\omega_{1}\right\} .
$$


If $\gamma_{1}=\sup \left\{\sup X\left(e_{0}, e_{1}\right): e_{0} \in S\right.$ and $\left.e_{1} \in S\left(e_{0}\right)\right\}$ and $Y\left(e_{0}, e_{1}\right)=X\left(e_{0}, e_{1}\right) \backslash \gamma_{1}$, then we have

$$
Y\left(e_{0}\right) \backslash \gamma_{1}=\bigcup\left\{Y\left(e_{0}, e_{1}\right): e_{1} \in S\left(e_{0}\right)\right\} \quad \text { for } e_{0} \in S .
$$

Let $\alpha\left(e_{0}, e_{1}\right)=\inf Y\left(e_{0}, e_{1}\right)$ and write $q\left(e_{0}, e_{1}\right)=p_{\alpha\left(e_{0}, e_{1}\right)}$. Continuing in this way we obtain a sequence $\gamma_{0}<\gamma_{1}<\gamma_{2}<\ldots$ of countable ordinals, a sequence $Y=\omega_{1}$, $Y\left(e_{0}\right), Y\left(e_{0}, e_{1}\right), \ldots$ of uncountable subsets of $\omega_{1}$, and subsets $S, S\left(e_{0}\right), S\left(e_{0}, e_{1}\right), \ldots$ of $[\omega]^{<\omega} \backslash\{\varnothing\}$ such that

$$
\omega_{1} \backslash \gamma_{0}=\bigcup\left\{Y\left(e_{0}\right): e_{0} \in S\right\}
$$

and for each $n \in \omega$

$$
Y\left(e_{0}, \ldots, e_{n}\right) \backslash \gamma_{n+1}=\bigcup\left\{Y\left(e_{0}, \ldots, e_{n+1}\right): e_{n+1} \in S\left(e_{0}, \ldots, e_{n}\right)\right\} .
$$

Define $T=\left\{\left\langle e_{0}, \ldots, e_{n}\right\rangle: e_{0} \in S, \ldots, e_{n} \in S\left(e_{0}, \ldots, e_{n-1}\right)\right\}$ and $\gamma=\sup \left\{\gamma_{n}: n \in \omega\right\}$. If $\alpha \geqslant \gamma$, then from the construction there are sets $e_{0} \in S, e_{1} \in S\left(e_{0}\right), e_{2} \in S\left(e_{0}, e_{1}\right), \ldots$ such that for all $n \in \omega, \alpha \in Y\left(e_{0}, \ldots, e_{n}\right)$. Thus $e=\left\langle e_{n}: n \in \omega\right\rangle$ is a branch of $T$ and

$$
p_{\alpha} * q\left(e_{0}, \ldots, e_{n}\right)=e_{n+1} \text {. }
$$

Also $e_{0}=p_{0} * p_{\alpha}$, so if we assume in addition $q(\varnothing)=\varnothing$, then $e_{n}=p_{\alpha} * q(e \mid n)$ also holds for $n=0$.

It remains to show that the finite nonempty sets $e_{n}$ are pairwise disjoint. Observe first that we have

$$
e_{n}=q(e \mid n) * p_{\alpha}=q(e \mid n) * q(e \mid m) \quad \text { for } m>n .
$$

Assume inductively that $e_{0}, \ldots, e_{n-1}$ are pairwise disjoint. We have

$$
e_{n}=\left(K_{q(e \mid n)} \cap L_{p_{\alpha}}\right) \cup\left(L_{q(e \mid n)} \cap K_{p_{\alpha}}\right) .
$$

Now, since $\bigcup L_{q(e \mid l)} \cap K_{p_{\alpha}} \subseteq K_{p_{\alpha}}$ and $K_{q(e \mid n)} \cap L_{p_{\alpha}} \subseteq L_{p_{\alpha}}$ and $K_{p_{\alpha}} \cap L_{p_{\alpha}}=\varnothing$ we have $\left(K_{q(e \mid n)} \cap L_{p_{\alpha}}\right) \cap\left(\bigcup_{i<n} L_{q(e \mid i)} \cap K_{p_{\alpha}}\right)=\varnothing$. And since $K_{q(e \mid n)} \cap L_{p_{\alpha}} \subseteq K_{q(e \mid n)}$ and $\bigcup_{i<n} K_{q(e \mid i)} \cap L_{p_{\alpha}} \subseteq L_{q(e \mid n)}$ and $K_{q(e \mid n)} \cap L_{q(e \mid n)}=\varnothing$, we have $\left(K_{q(e \mid n)} \cap L_{p_{\alpha}}\right)$ $\cap\left(\bigcup_{i<n}^{i<n} K_{q(e \mid i)} \cap L_{p_{\alpha}}\right)=\varnothing$. It follows that $\left(K_{q(e \mid n)} \cap L_{p_{\alpha}}\right) \cap\left(e_{0} \cup \ldots \cup e_{n-1}\right)=\varnothing$. Symmetrically, $L_{q(e \mid n)} \cap K_{p_{s}}$ is disjoint from $e_{0}, \ldots, e_{n-1}$ and the proof is complete.

Remark. Let $\bar{\gamma}$ exceed the domains of all the conditions $p_{\alpha}, \alpha<\gamma$. Then the branch $e=\left\langle e_{n}: n \in \omega\right\rangle$ corresponding to any $p_{\alpha}$ with $\alpha \geqslant \gamma$ can be defined from the following parameters: $T, q$ and the sets $\left\{A_{\beta}: \beta<\bar{\gamma}\right\} ; p_{\alpha}$ and the sets

$$
\left\{A_{\beta}: \beta \in \operatorname{Dm}\left(s_{p_{\alpha}}\right) \cup \operatorname{Dm}\left(t_{p_{\alpha}}\right)\right\} .
$$

3. As was remarked earlier, the separating forcings $\boldsymbol{P}_{E}\left\{A_{\alpha}\right\}$, which we intend to iterate, need not satisfy the c.c.-condition. Here we prove that any such $\boldsymbol{P}_{\boldsymbol{E}}\left\{A_{\alpha}\right\}$ has an improvement; in fact, an improvement will be produced in the course of iteration in at most $\omega_{1}$ steps. Hence we consider below a finite support iteration
$\boldsymbol{P}=\sum_{\alpha<\omega_{1}} \boldsymbol{P}_{\alpha}$, which should be understood as a fragment of length $\omega_{1}$ of our "real" iteration described in Section 4.

LEMMA. Let $\boldsymbol{P}=\sum_{\alpha<\omega_{1}} \boldsymbol{P}_{\alpha}$ be a finite support iteration of nontrivial forcings satisfying the c.c.-condition and let $G \subseteq \boldsymbol{P}$ be a generic filter over $V$. If an almost disjoint family $\left\{A_{\alpha}: \alpha<\omega_{1}\right\}$ and $a$ set $E \subseteq \omega_{1}$ are in $V$, then for each family $\left\{F_{\alpha}: \alpha<\omega_{1}\right\}$ of ultrafilters in $V[G]$ such that $A_{\alpha} \in F_{\alpha}$, there are sets $B_{\alpha} \subseteq A_{\alpha}$ in $V[G], B_{\alpha} \in F_{\alpha}$, such that $\boldsymbol{P}_{E}\left\{B_{\alpha}\right\}$ is an improvement of $\boldsymbol{P}_{E}\left\{A_{\alpha}\right\}$ (i.e. $\boldsymbol{P}_{E}\left\{B_{\alpha}\right\}$ satisfies the c.c.-condition in $V[G]$ ).

Proof. It is well known that a finite support iteration of nontrivial forcings adds a Cohen set $c \subseteq \omega$ in each sequence of $\omega$ steps. Thus let $\lambda_{\alpha}$ be an increasing enumeration of all countable limit ordinals and let $c_{\alpha} \in V\left[G_{\lambda_{\alpha+1}}\right]$ be a Cohen set over $V\left[G_{\lambda_{\alpha}}\right]$. Define

$$
B_{\alpha}= \begin{cases}A_{\alpha} \cap c_{\alpha} & \text { if } c_{\alpha} \in F_{\alpha}, \\ A_{\alpha} \backslash c_{\alpha} & \text { if } c_{\alpha} \notin F_{\alpha} .\end{cases}
$$

Thus $B_{\alpha} \subseteq A_{\alpha}$ and $B_{\alpha} \in F_{\alpha}$ for each $\alpha<\omega_{1}$. It remains to show that $\boldsymbol{P}_{E}\left\{\boldsymbol{B}_{\alpha}\right\} \in V[G]$ satisfies the c.c.-condition $V[G]$. Assume, on the contrary that there is an uncountable antichain $\left\{p_{\alpha}: \alpha<\omega_{1}\right\}$ in $\boldsymbol{P}_{E}\left\{B_{\alpha}\right\}$ (with the properties described in Section 2). Usually, a condition from $\boldsymbol{P}_{E}\left\{B_{\alpha}\right\}$ need not be in $\boldsymbol{P}_{E}\left\{A_{\alpha}\right\}$ but since the domains of the conditions in the antichain are pairwise disjoint, for each $\alpha<\omega_{1}$ there is a $k_{\alpha} \in \omega$ such that if

and

$$
\tilde{A}_{\gamma}=\left(B_{\gamma} \cap k_{\alpha}\right) \cup\left(A_{\gamma} \backslash k_{\alpha}\right) \quad \text { for } \gamma \in \operatorname{Dm}\left(s_{p_{\alpha}}\right) \cup \operatorname{Dm}\left(t_{p_{\alpha}}\right)
$$

$$
\tilde{A}_{\gamma}=B_{\gamma} \quad \text { for } \gamma \notin \bigcup_{\alpha<\omega_{1}} \operatorname{Dm}\left(s_{p_{\alpha}}\right) \cup \operatorname{Dm}\left(t_{p_{\alpha}}\right)
$$

then each $p_{\alpha}$ is a condition in $\boldsymbol{P}_{E}\left\{\tilde{A}_{\gamma}\right\}$. Moreover, $\left\{p_{\alpha}: \alpha<\omega_{1}\right\}$ is then an antichain in $\boldsymbol{P}_{E}\left\{\tilde{A}_{\gamma}\right\}$ because $p_{\alpha} * p_{\beta}$ calculated in $\boldsymbol{P}_{E}\left\{\boldsymbol{B}_{\gamma}\right\}$ is a subset of $p_{\alpha} * p_{\beta}$ calculated in $\boldsymbol{P}_{E}\left\{\tilde{A}_{\gamma}\right\}$, and hence $p_{\alpha} * p_{\beta} \neq \varnothing$ in $\boldsymbol{P}_{E}\left\{\tilde{A}_{\gamma}\right\}$. We now apply the Lemma of Section 2 to the antichain $\left\{p_{\alpha}: \alpha<\omega_{1}\right\}$ in $\boldsymbol{P}_{E}\left\{A_{\alpha}\right\}$ and take a $\beta<\omega_{1}$ such that $T, \gamma, q$, $\left\{A_{\xi}: \xi<\bar{\gamma}\right\}$ (cf. Remark at the end of Section 2) are all in $V\left[G_{\beta}\right]$. Fix an $\alpha>\beta$ and let $\gamma_{1}<\ldots<\gamma_{r}$ enumerate $\operatorname{Dm}\left(s_{p_{\alpha}}\right) \cup \operatorname{Dm}\left(t_{p_{\alpha}}\right)$. Since $p_{\alpha}$ and $A_{\gamma_{1}}, \ldots, A_{\gamma_{r}}$ are in $V$, we infer that the branch $e=\left\langle e_{n}: n \in \omega\right\rangle$ corresponding to $p_{\alpha}$ belongs to $V\left[G_{\beta}\right]$. Since $\beta<\alpha \leqslant \gamma_{1} \leqslant \lambda_{\gamma_{1}}$, the set $c_{\gamma_{1}}$ is a Cohen set over $V\left[G_{\beta}\right]$, and hence the set

$$
S_{\gamma_{1}} e=\left\{e_{n}: e_{n} \subseteq S_{\gamma_{1}}\right\}
$$

is infinite, where $S_{\gamma}$ denotes $c_{\gamma}$ if $c_{\gamma} \in F_{\gamma}$ and $\omega \backslash c_{\gamma}$ otherwise. After $r$ steps we obtain an infinite family $S_{\gamma_{r}} \ldots S_{\gamma_{1}} e \subseteq\left\{e_{n}: n \in \omega\right\}$. Choose an $e_{n}$ from $S_{\gamma_{r}} \ldots S_{\gamma_{1}} e$. By the definition of the $B_{\gamma}$ 's we see that

$$
B_{\gamma} \subseteq A_{\gamma} \backslash e_{n} \quad \text { for } \gamma=\gamma_{1}, \ldots, \gamma_{r},
$$

and hence $p_{\alpha} * q(e \mid n)$ in $\boldsymbol{P}_{\boldsymbol{E}}\left\{B_{\gamma}\right\}$ is disjoint from $e_{n}$. On the other hand, $p_{\alpha} * q(e \mid n)$ in $P_{E}\left\{B_{\gamma}\right\}$ is a subset of $p_{\alpha} * q(e \mid n)$ in $\boldsymbol{P}_{E}\left\{\tilde{A}_{\gamma}\right\}$, i. e. a subset of $e_{n}$. Hence $p_{\alpha} * q(e \mid n)=\varnothing$, 2- Fundamenta Mathematicae 129, 3 
which means that $p_{\alpha}$ and $q(e \mid n)$ are compatible, a contradiction. The proof of the lemma is complete.

4. Now, we can finish the proof of our main theorem. We begin with $V=L$ and shall use the following principle $\diamond$ : there is a sequence $\left\langle S_{\alpha}: \alpha<\omega_{2}\right.$ and $\left.\operatorname{cf}(\alpha)=\omega_{1}\right\rangle$ such that for each $X \subseteq \omega_{2}$, the set $\left\{\alpha: X \cap \alpha=S_{\alpha}\right\}$ is stationary. Let $H$ be the family of all sets (in $V$ ) of hereditary power $<\omega_{2}$ and $f: \omega_{2} \rightarrow H$ a bijection. Set $H_{\alpha}=f[\alpha]$ for each $\alpha$, and $T_{\alpha}=f\left[S_{\alpha}\right]$ whenever $\operatorname{cf}(\alpha)=\omega_{1}$. Then, for each $Y \subseteq H$, the set $\left\{\alpha: Y \cap H_{\alpha}=T_{\alpha}\right\}$ is stationary.

Each forcing $\boldsymbol{P}_{\alpha}$ defined below is of cardinality $\leqslant \omega_{1}$, and hence $\boldsymbol{P}_{\alpha}$-names under consideration can be regarded as elements of the set $H$.

Let $\boldsymbol{P}_{0}=$ the Cohen forcing and $\boldsymbol{P}_{\alpha}=\sum_{\beta<\alpha} \boldsymbol{P}_{\beta}$ (the direct limit), for each limit $\alpha<\omega_{2}$. If $\operatorname{cf}(\alpha)=\omega_{1}$ we look at $T_{\alpha}$ and if $\boldsymbol{P}_{\alpha} \Vdash$ " $T_{\alpha}$ satisfies the c.c.-condition" then let $\boldsymbol{P}_{\alpha+1}=\boldsymbol{P}_{\alpha} * T_{\alpha}$; and if $T_{\alpha}$ is a disjoint union $T_{\alpha}=a \cup e \cup d$ of $\boldsymbol{P}_{\alpha}$-names of an almost disjoint family, of a subset of $\omega_{1}$ and of a strongly discrete set of ultrafilters, respectively, and $\boldsymbol{P}_{\alpha}$ t "there is an improvement of $\boldsymbol{P}_{e}\{a\}$ w.r.t. $d$ " then let $\boldsymbol{P}_{\alpha+1}=\boldsymbol{P}_{\alpha} * Q$ where $Q$ is a $\boldsymbol{P}_{\alpha}$-name of such an improvement. Finally, let $\boldsymbol{P}_{\alpha+1}=\boldsymbol{P}_{\alpha}$ in each remaining case.

Let $\boldsymbol{P}=\sum_{\alpha<\omega_{2}} \boldsymbol{P}_{\alpha}$ and let $G \subseteq \boldsymbol{P}$ be a generic filter.

Obviously, in $V[G]$ Martin's Axiom plus $2^{\omega_{0}}=\omega_{2}$ hold true. Now, let $D=\left\{F_{\alpha}: \alpha<\omega_{1}\right\} \in V[G]$ be a strongly discrete sequence of ultrafilters. Fix an almost disjoint family $\left\{A_{\alpha}: \alpha<\omega_{1}\right\}$ with $A_{\alpha} \in F_{\alpha}$ and a subset $E \subseteq \omega_{1}$. Then, for some $\beta<\omega_{2}$, both $\left\{A_{\alpha}: \alpha<\omega_{1}\right\}$ and $E$ are in $V\left[G_{\beta}\right]$. The restricted sequence $D \mid \gamma=\left\{F_{\alpha} \cap V\left[G_{\gamma}\right]: \alpha<\omega_{1}\right\}$ need not belong to $V\left[G_{\gamma}\right]$ but it does for many $\gamma$ 's: the set $\left\{\gamma<\omega_{2}: D \mid \gamma \in V\left[G_{\gamma}\right]\right\}$ is $\omega_{1}$-normal (i.e. it is unbounded in $\omega_{2}$ and closed under $\omega_{1}$-limits).

To see this let us encode $D$ as

$$
D=\left\{\langle\alpha, x\rangle: \alpha<\omega_{1} \text { and } x \in F_{\alpha}\right\}
$$

Then the restrictions are of the form

$$
D \mid \gamma=\left\{\langle\alpha, x\rangle \in D: x \in V\left[G_{\gamma}\right]\right\} .
$$

We choose a canonical $\boldsymbol{P}$-name $\underline{D}$ for $D$ which consists of pairs $\left\langle\langle\alpha, x\rangle^{\boldsymbol{p}}, p\right\rangle$, where $x$ is a canonical name for $x \subseteq \omega, p \in P$ and

$$
\underline{D}(\alpha, x)=\left\{p \in \boldsymbol{P}:\left\langle\langle\alpha, x\rangle^{\boldsymbol{P}}, p\right\rangle \in \underline{D}\right\}
$$

is an antichain. Define the subnames $\underline{D} \mid \gamma$ :

$$
\underline{D} \mid \gamma=\left\{\left\langle\langle\alpha, \underline{x}\rangle^{\boldsymbol{P}}, p\right\rangle \in \underline{D}: \underline{x} \in V^{\boldsymbol{P}_{\gamma}} \text { and } \underline{D}(\alpha, x) \subseteq \boldsymbol{P}_{\gamma}\right\} \text {. }
$$

Then $\underline{D} \mid \gamma$ is a $\boldsymbol{P}_{\gamma}$-name. The set

$$
C_{1}=\left\{\gamma<\omega_{2}: \forall x, \alpha\left[x \in V^{\boldsymbol{P}_{\gamma}} \rightarrow \underline{D}(\alpha, x) \subseteq \boldsymbol{P}_{\gamma}\right]\right\}
$$

is $\omega_{1}$-normal and for $\gamma \in C_{1}$ we have

$$
(\underline{D} \mid \gamma)\left[G_{\gamma}\right]=D \mid \gamma,
$$

and thus $D \mid \gamma \in V\left[G_{\gamma}\right]$ for each $\gamma \in C_{1}$. Note that then $D \mid \gamma$ is a strongly discrete sequence of ultrafilters in $V\left[G_{\gamma}\right]$.

Now we take $\boldsymbol{P}_{\beta}$-names $a$ and $e$ for $\left\{A_{\alpha}: \alpha<\omega_{1}\right\}$ and $E$, respectively. Since $a$ and $e$ are in $H$ and $\underline{D} \subseteq H$, it is easy to check that the set

$$
C_{2}=\left\{\gamma<\omega_{2}:(a \cup e \cup \underline{D}) \cap H_{\gamma}=a \cup e \cup(\underline{D} \mid \gamma)\right\}
$$

is $\omega_{1}$-normal. Let $C=C_{1} \cap C_{2}$. Applying the principle $\diamond$ and the lemma of the preceding section, we can find a large enough $\gamma \in C$, with $\operatorname{cf}(\gamma)=\omega_{1}$, for which $T_{\gamma}=a \cup e \cup(\underline{D} \mid \gamma)$ and $\boldsymbol{P}_{\gamma} \Vdash$ "there is an improvement of $\boldsymbol{P}_{e}\{a\}$ w.r.t. $\underline{D} \mid \gamma$ ". Hence $\boldsymbol{P}_{\gamma+1}=\boldsymbol{P}_{\gamma} * Q$ for a $\boldsymbol{P}_{\gamma}$-name $Q$ of such an improvement. Thus in $V\left[\boldsymbol{G}_{\gamma+1}\right]$ there are sets $A, B \subseteq \omega$ such that in $V[G]$ we have

$$
\left\{F_{\alpha}: \alpha \in E\right\} \subseteq A^{*}, \quad\left\{F_{\alpha}: \alpha \notin E\right\} \subseteq B^{*} \quad \text { and } \quad A^{*} \cap B^{*}=\varnothing,
$$

which finishes the proof of the theorem.

If we wish to conclude, in addition, that $\beta \omega_{1}$ is not a continuous image of $\omega^{*}$ we have to combine our forcing with that in Frankiewicz [1985]. Let $B$ denote the Boolean algebra contained in $P\left(\omega_{1}\right)$ generated by countable subsets of $\omega_{1}$. We add one more case in the iteration: if $\operatorname{cf}(\alpha)=\omega_{1}$ and $\boldsymbol{P}_{\alpha} \Vdash$ " $T_{\alpha}$ is an embedding of $B$ into $P(\omega) /$ fin" then let $\boldsymbol{P}_{\alpha+1}=\boldsymbol{P}_{\alpha} * Q$, where $Q$ is a $\boldsymbol{P}_{\alpha}$-name of a c.c.c. forcing making the gap $T_{\alpha}(L)$, for an $L$ in $B$, indestructible.

5. We conclude the paper with some simple remarks on $B$-ideals. We include these remarks here since the method used below is very similar to that in Section 2 .

A nonprincipal ideal $J$ over $\omega$ is called a $B$-ideal if the following holds: whenever the sets $A_{n}$ are in $J$ and

$$
\min _{n} A_{n} \rightarrow \infty
$$

then, for some infinite $z \subseteq \omega, \bigcup\left\{A_{n}: n \in Z\right\} \in J$.

Burzyk [198•] uses such ideals to construct certain normed linear spaces. Observe that (the dual of) a $P$-point is a $B$-ideal. Indeed, if $A_{n} \in J$, then there is an $A \in J$ such that $A_{n} \subseteq{ }_{*} A$ for each $n \in \omega$. Writing $e_{n}=A_{n} \backslash A$ we have, for any $Z \subseteq \omega$,

$$
\bigcup\left\{A_{n}: n \in Z\right\} \subseteq A \cup \cup\left\{e_{n}: n \in Z\right\} .
$$

If $\min A_{n} \rightarrow \infty$, then we can find a $Z \subseteq \omega$ such that $\left\{e_{n}: n \in Z\right\}$ is a disjoint family. For any partition $Z=Z_{0} \cup Z_{1}$, the sets

$$
\bigcup\left\{e_{n}: n \in Z_{0}\right\} \text { and } \bigcup\left\{e_{n}: n \in Z_{1}\right\}
$$

are disjoint and hence one of them is in $J$; denote it by $Y$. Then we have

$$
\bigcup\left\{A_{n}: n \in Y\right\} \subseteq A \cup \cup\left\{e_{n}: n \in Y\right\} \in J
$$

and hence $\left\{A_{n}: n \in Y\right\}$ is in $J$.

$2^{*}$ 
Thus the existence of $B$-ideals follows, for example, from the Continuum Hypothesis. Now, it is easy to see that each $B$-ideal is a $P$-ideal (but not necessarily maximal). Indeed, suppose that $A_{n} \in J$, where $J$ is a $B$-ideal. We may assume that the sequence is increasing. If $B_{n}=A_{n} \backslash n$, then the $B_{n}$ 's are in $J$ and $\min B_{n} \rightarrow \infty$, and hence, for some $Z, B=\bigcup\left\{B_{n}: n \in Z\right\}$ is in $J$. But $A_{n} \subseteq{ }_{*} B_{n} \subseteq B$ " for each $n \in \omega$, and hence $J$ is a $P$-ideal.

Finally, we prove the following

Proposition. Assuming $\mathrm{CH}$, there is a $B$-ideal, and hence a $P$-ideal, which cannot be extended to a $P$-point. In particular, there are nonmaximal $B$-ideals.

Proof. The Balcar-Frankiewicz-Mills Theorem shows that the space $G\left(2^{\omega}\right)$ (the Gleason space of the Cantor set) can be embedded into $\omega^{*}$ as a closed $P$-set $X$. Hence the family

$$
F=\left\{A \subseteq \omega: X \subseteq A^{*}\right\}
$$

is a $P$-filter. If $F$ were extendible to a $P$-point $p$ then, since $\{p\}=\bigcap\left\{A^{*}: A \in p\right\}$ and $A \cap X \neq \varnothing$ for each $A \in p$, we would have $p \in X$, which is impossible, because $X$ is separable and without isolated points. The dual $J=\{\omega \backslash A: A \in F\}$ is then a $P$-ideal not extendible to a $P$-point and, in fact, it is a $B$-ideal: suppose that $A_{n} \in J$ and $\min A_{n} \rightarrow \infty$. Let $A \in J$ almost contain each $A_{n}$ and let $e_{n}=A_{n} \backslash A$. There is an infinite $Z \subseteq \omega$ such that $\left\{e_{n}: n \in Z\right\}$ is a disjoint family. It is possible to form $2^{\omega}$ almost disjoint subunions $\bigcup\left\{e_{n}: n \in Z_{\alpha}\right\}$, for almost disjoint $Z_{\alpha} \subseteq Z$. One of them is in $J$, for otherwise we would have $2^{\omega}$ nonempty open-closed disjoint subsets of $X$, which is impossible as $\boldsymbol{G}\left(2^{\omega}\right)$ has countable cellularity.

\section{References}

19801 B. Falcar, R. Frankiewicz and C. B. Mills, More On Nowhere Dense Closed P-sets Bull. Acad. Pol. Sci. 28, 295-299.

[1981] B. Balcar, P. Simon and P. Vojtás, Refinement Properties and Extensions of Filters in Boolean Algebras, Trans. Amer. Math. Soc. 267, 265-283.

[198 .] J. Burzyk, An Example of a Noncomplete N-Space, to appear.

[1968] R. Engelking, Outline of General Topology, PWN-North Holland, Warszawa-Amsterdam 1968.

[1985] R. Frankiewicz, Some remarks on embedding of boolean algebras and topological spaces, $\mathrm{Mr}$. Fund. Math. 126, 63-68.

Received 27 January 1986;

in revised form 20 August 1986

\section{Nielsen reduction in free groups with operators}

by

\author{
Gert Denk and Wolfgang Metzler (Frankfurt)
}

Abstract. The Nielsen method is generalized to an equivariant situation, in which the variables of a free group are freely permuted by an operator group $G$. Critical elements $W=A \cdot x(A)^{-1}$, $x \in G$ ocur, which are andusho-Neumann Theorem is deduced and applications to low-dimensional CW-complexes are given.

§ I. Introduction. Let $G$ be an arbitrary group, $F\left(a_{1}, \ldots, a_{n}\right)$ a free group of finite rank, and let $\vec{F}$ be the normal closure of $F$ in $G * F . \bar{F}$ is freely generated by the $x a_{i} x^{-1}, x \in G$, with $G$ operating on $\bar{F}$ by conjugation. Alternatively we may think of $F$ as a free group with basis $x\left(a_{i}\right)\left(\hat{\triangleq} x a_{i} x^{-1}\right), x \in G$, which is freely permuted by $G$. The length of an element $W$ of $\bar{F}$ is understood to be the length with respect to the (in general infinite) basis $x\left(a_{i}\right)$ and is denoted by $|W|$.

If $W_{1}, \ldots, W_{m}$ are finitely many elements of $\bar{F}$, then we denote by $\mathrm{Gp}\left(W_{1}, \ldots, W_{m}\right)$ the subgroup of $\bar{F}$ generated by the $W_{i}$; by $\overline{\mathrm{Gp}\left(W_{1}, \ldots, W_{m}\right)}$ we denote the smallest $G$-invariant subgroup of $\bar{F}$ containing the $W_{i}$, i.e. the subgroup, which is generated by all $x\left(W_{i}\right), x \in G .\left(W_{1}, \ldots, W_{m}\right)$ is called a $G$-generating system of $\overline{\mathrm{Gp}\left(W_{1}, \ldots, W_{m}\right)} \cdot A G$-generating system is called $(G-)$ free or a $(G$-) basis of $\left.\frac{\mathrm{of}}{\mathrm{Gp}\left(W_{1}, \ldots,\right.}, \ldots, W_{m}\right)$, if the $x\left(W_{i}\right), x \in G, i=1, \ldots, m$ are free in the ordinary sense. If a $G$-invariant subgroup of $\bar{F}$ has a $G$-basis, then this subgroup is said to be $G$-free.

$\mathrm{Gp}\left(W_{1}, \ldots, W_{m}\right)$ remains unchanged if the $m$-tuple $\left(W_{1}, \ldots, W_{m}\right)$ is subject to Nielsen transformations (NT), i.e. a finite sequence of the following elementary transformations:

(i) $W_{i} \rightarrow W_{i}^{-1}$ for some $i$ (inversion),

(1) (ii) $W_{i} \rightarrow W_{i} W_{j}, i \neq j$ (multiplication),

(iii) deletion of some $W_{i}$, where $W_{i}=1$.

For $\overline{\mathrm{Gp}} \overline{\left(W_{1}, \ldots, W_{m}\right)}$ we may enlarge this list by

(2) (iv) $W_{t} \rightarrow x\left(W_{i}\right)$ for some $i, x \in G((G-)$ conjugation) 